\title{
Alterstice
}

Revue internationale de la recherche interculturelle

International Journal of Intercultural Research

Revista International de la Investigacion Intercultural

\section{Réponses institutionnelles à la souffrance psychique des personnes requérantes d'asile : l'expérience genevoise}

\section{Javier Bartolomei, Rachel Baeryswil-Cottin et Natacha Premand}

Volume 4, numéro 2, 2014

Santé mentale et sociétés plurielles

URI : https://id.erudit.org/iderudit/1077427ar

DOI : https://doi.org/10.7202/1077427ar

Aller au sommaire du numéro

Éditeur(s)

Alterstice

ISSN

1923-919X (numérique)

Découvrir la revue

Citer cet article

Bartolomei, J., Baeryswil-Cottin, R. \& Premand, N. (2014). Réponses

institutionnelles à la souffrance psychique des personnes requérantes d'asile : l'expérience genevoise. Alterstice, 4(2), 77-84. https://doi.org/10.7202/1077427ar
Résumé de l'article

La multiplication et l'intensification des flux migratoires des dernières années ont conduit à un afflux parfois soudain de nouvelles populations venant frapper à nos portes pour demander asile. Si le parcours migratoire de ces personnes peut se ressembler, leur histoire, leurs trajectoires et de ce fait leurs besoins varient et viennent par là-même interroger notre système de soins et ses capacités d'adaptation. L'hétérogénéité des tableaux cliniques rencontrés nous oblige dès lors à proposer différentes modalités de réponses institutionnelles à la souffrance psychique de cette population, qui tiennent compte à la fois de ses références culturelles, de la clinique du trauma dont elle peut être porteuses, mais aussi de la part de souffrance liée à la violence des conditions d'accueil qu'elle peut rencontrer. 
ARTICLE THÉMATIQUE

\title{
Réponses institutionnelles à la souffrance psychique des personnes requérantes d'asile : l'expérience genevoise
}

\author{
Javier Bartolomei ${ }^{1}$, Rachel Baeryswil-Cottin ${ }^{1}$ et Natacha Premand ${ }^{1}$
}

\section{Résumé}

La multiplication et l'intensification des flux migratoires des dernières années ont conduit à un afflux parfois soudain de nouvelles populations venant frapper à nos portes pour demander asile. Si le parcours migratoire de ces personnes peut se ressembler, leur histoire, leurs trajectoires et de ce fait leurs besoins varient et viennent par làmême interroger notre système de soins et ses capacités d'adaptation. L'hétérogénéité des tableaux cliniques rencontrés nous oblige dès lors à proposer différentes modalités de réponses institutionnelles à la souffrance psychique de cette population, qui tiennent compte à la fois de ses références culturelles, de la clinique du trauma dont elle peut être porteuses, mais aussi de la part de souffrance liée à la violence des conditions d'accueil qu'elle peut rencontrer.

\section{Rattachement de l'auteur}

${ }^{1}$ Hôpitaux universitaires de Genève, Genève, Suisse

\section{Correspondance}

javier.bartolomei@hcuge.ch

\section{Mots clés}

Psychiatrie transculturelle; soins psychiatriques multidisciplinaires; patients requérants d'asile

\section{Pour citer cet article}

Bartolomei, J., Baeryswil-Cottin, R. et Premand, N. (2014). Réponses institutionnelles à la souffrance psychique des personnes requérantes d'asile : l'expérience genevoise. Alterstice, 4(2), 77-84. 


\section{Introduction}

Ces dernières années, l'accueil des personnes en demande d'asile a suscité d'intenses débats en Suisse et a fait l'objet de nombreuses votations (Sanchez Mazas, 2011). Dans un climat parfois passionné, le risque est grand de céder à une vision caricaturale de la question de l'octroi d'asile et de passer sans transition d'une image idéalisée du " bon requérant d'asile ", en détresse et démuni, à celle diabolisée de l'étranger en quête de bénéfices sociaux (D'Halluin, 2006). Voilà un défi permanent pour nous, soignants, de pouvoir, au-delà de nos projections, proposer un accueil et des soins qui tiennent compte de toute la singularité des parcours migratoires et de la diversité des références culturelles rencontrées.

En effet, notre travail quotidien auprès de cette population nous confronte à une grande hétérogénéité de formes de souffrance mentale, et même s'il existe des facteurs de crise qui se répètent (refus d'octroi d'asile, avis d'expulsion notamment), chaque personne requérante d'asile y réagit différemment. De ce fait, notre programme de soins psychiatriques transculturels vise à pouvoir proposer plusieurs réponses institutionnelles à ces divers tableaux cliniques, et il est sous-tendu par une volonté d'adapter au mieux notre offre de soins. Si certains patients peuvent par exemple eux-mêmes formuler une demande d'aide, d'autres y seront fortement réticents malgré une intense souffrance (Mc Coll et Johnson, 2006), du fait de leur psychopathologie ou de barrières culturelles. Comment dès lors s'approcher de cette souffrance en tenant compte de leurs besoins et de leurs appréhensions ? Quelle réponse donner au vécu d'exclusion dont ils sont parfois porteurs ? Voilà différentes questions auxquelles nous nous efforcerons dans cet article d'apporter des éléments de réponse découlant de notre expérience clinique.

\section{Organisation des soins psychiques proposés aux requérants d'asile à Genève}

Probablement en lien avec une forte tradition de métissage culturel vieille de plusieurs centaines d'années à Genève, la psychiatrie genevoise s'intéresse depuis longtemps à la thématique de la santé mentale des personnes migrantes. Ainsi, la première consultation institutionnelle de psychiatrie transculturelle a vu le jour voilà une trentaine d'années, soutenue en parallèle par l'apparition de diverses structures à vocation sociale ou prodiguant des soins psychologiques (Premand et collab., 2013). Aujourd'hui, lorsqu'une personne requérante d'asile arrive à Genève, elle se voit attribuer un médecin généraliste, installé en cabinet ou travaillant en institution. Les frais d'assurance maladie sont pris en charge par l'État et les requérants d'asile sont assurés d'emblée à leur arrivée. Le médecin de premier recours peut par délégation diriger cette personne vers une consultation publique de psychiatrie, en l'occurrence notre programme de psychiatrie transculturelle. En effet, ce dernier propose des soins psychiatriques institutionnels multidisciplinaires à l'ensemble des personnes requérantes d'asile vivant dans les foyers gérés par les services sociaux de la ville. À relever que cette consultation fait partie d'une structure psychiatrique qui prodigue parallèlement des soins aux résidents genevois vivant dans la zone géographique qu'elle dessert, dans une logique de soins de proximité de type consultation de secteur ${ }^{1}$. Nos soins aux personnes requérantes découlent principalement d'une approche de type psychiatrie communautaire et sociale, privilégiant le travail de réseau avec différents interlocuteurs du monde juridique et social et s'adressant notamment à une population souffrant de graves troubles psychiques ne pouvant bénéficier d'un abord psychothérapeutique stricto sensu. D'autres organismes de type associatifs proposent des approches de type psychothérapeutique individuelles ou groupales à une frange de personnes migrantes ayant subi des violences dans leur pays d'origine, mais détentrice en majorité d'un permis de séjour.

\section{Modalités de réponse institutionnelle à la souffrance mentale des personnes requérantes d'asile}

Nos interventions sont principalement de trois types et découlent des modalités d'intervention usuelle de la psychiatrie sociale et communautaire. Elles regroupent :

- des interventions de crise (concernent $20 \%$ de la cohorte soignée),

\footnotetext{
${ }^{1}$ L'ensemble des caractéristiques de la cohorte des patients requérants d'asile suivis dans notre centre de soins est disponible dans l'article de Premand et collab. (2013).
} 
- des prises en soins multidisciplinaires continues sur le moyen et long terme (modalité de suivi de $70 \%$ de la cohorte soignée),

- des interventions de type "équipe mobile », notamment dans les différents foyers où séjournent les patients requérants d'asile (10\% de la cohorte).

Ces trois types d'intervention correspondent à des " temps cliniques " différents d'une part, mais aussi à des sousgroupes distincts de cette population susceptibles d'avoir des besoins spécifiques.

\section{Interventions de type " crise »}

Les interventions de type crise (d'une durée habituelle de 2 à 4 mois) constituent souvent la porte d'entrée dans les soins psychiatriques pour ces patients. Elles impliquent la mobilisation rapide d'une équipe multidisciplinaire et ont la particularité d'être fortement imprégnées par un modèle de référence psychanalytique qui a représenté l'une des premières théorisations de l'intervention de crise à Genève (De Coulon et Von Oberbeck Ottino, 1999). La notion de réactualisation d'un traumatisme initial par un facteur de crise récent y est centrale. Transposé à la situation des patients requérants d'asile, ce facteur de crise prend principalement la forme du rejet d'une demande d'octroi d'asile ou d'un déménagement dans un nouveau foyer, souvent synonyme d'une avancée dans le processus d'expulsion. Ce type d'événement pourra avoir diverses résonances et renvoyer par exemple à :

- un sentiment de non-reconnaissance des traumas subis et donc à une remise en question du statut de victime, potentiellement associé à la crainte de subir de nouvelles agressions dans le pays d'origine,

- un sentiment d'échec du projet migratoire, ressenti qui n’est pas spécifique des requérants d'asile,

- un risque de nouveau déracinement, si le patient vit depuis plusieurs années en Suisse et y a créé des liens et s'il se voit renvoyé dans un pays qui lui est devenu étranger,

- un sentiment d'injustice si d'autres membres de sa famille ou de sa communauté sont autorisés à rester dans le pays,

- une désillusion vis-à-vis de la Suisse comme pays des droits de l'Homme, de la Croix-Rouge et des grandes organisations internationales.

À travers une symptomatologie principalement anxieuse et dépressive, parfois accompagnée d’idéations suicidaires, c'est non seulement une clinique du trauma et de sa réactivation qui peut être au premier plan, mais également une clinique de la déception. La première est probablement la plus connue et la plus documentée (Steel et collab., 2009) : le traumatisme, par son intensité, dépasse les capacités de représentation du sujet et finit par le hanter, à travers les flash-back, les cauchemars ou les réactions d'hypervigilance. Cette clinique peut faire écho à des événements traumatiques survenus tôt dans l'histoire du sujet, et pourtant oubliés de lui-même. II est cependant souvent difficile de pouvoir remonter le fil de l'histoire de ces patients jusqu'aux relations précoces ou au vécu de la petite enfance, tant les événements du passé récent (menace de renvoi, traumatismes vécus dans le pays d'origine avant le parcours migratoire) peuvent faire écran. Les perturbations de l'axe temporel et de la mémoire renvoient à une rupture du sentiment d'identité (Sironi, 1999), bloquant la reconstruction d'un sentiment de continuité du sujet après le ou les événements traumatiques. Si nous faisons parfois l'hypothèse de carences affectives ou d'abus survenus dans l'enfance réactivés par la procédure d'asile et le sentiment d'insécurité à laquelle elle renvoie, nous ne pouvons que rarement l'étayer par un récit anamnestique détaillé.

À cette clinique du trauma peut s'ajouter une clinique de la désillusion, de la déception, où la thématique de la non-reconnaissance prédomine, qui trouve son acmé lorsque le patient prend connaissance du rejet de sa demande d'asile. Pour tous les sujets migrants, la réussite du projet migratoire est un déterminant important de la santé mentale. Pour les requérants d'asile, l'octroi du droit d'asile peut de manière plus spécifique permettre de se dégager d'une histoire traumatique en lui donnant un sens : la possibilité d'une nouvelle vie dans un pays d'accueil.

De ce fait, les tableaux cliniques que nous rencontrons lors des interventions de crise résultent souvent de la mise sous tension de ces deux cliniques, celle du trauma et celle de la déception. Chacune peut avoir un effet synergique l'une sur l'autre. II en résulte des présentations psychopathologiques où prédominent les troubles de l’humeur, 
parfois accompagnés de symptômes pouvant évoquer un trouble de la personnalité, tels que des scarifications, des tentamens médicamenteux à répétition ou une intolérance à la frustration. Néanmoins, souvent sans données anamnestiques pré-migratoires consistantes, il demeure difficile de déterminer si des traits de personnalité pathologiques sont préexistants au vécu traumatique (et ont été plus ou moins accentués par celui-ci) ou si leur présence résulte $d$ 'un remodèlement de la personnalité survenu après ces événements. Concernant la première hypothèse, des auteurs admettent que certains traits de personnalités pourraient favoriser l'émergence d'un syndrome de type des troubles de stress post-traumatique (PTSD) (Williams, 1999). Ainsi, l'étude de Hovela et Tarrier (2001) relève des corrélations significatives entre des scores élevés sur la dimension " neuroticisme " (tendance à éprouver des émotions négatives, instabilité émotionnelle, faible capacité d'adaptation [coping]) et le développement d'une symptomatologie de type PTSD après la survenue d'un traumatisme. La seconde hypothèse serait soutenue par une étude de Hunt et Gakenyi (2005) comparant l'état mental de Bosniaques réfugiés et celui de Bosniaques déplacés dans leur propre pays, et qui révèle chez les premiers des scores de trauma significativement plus élevés ainsi que des traits de personnalité évitante plus fréquents (tendances aux anticipations anxieuses négatives, crainte de l'incertitude, timidité et fatigabilité), traits fréquemment associés aux troubles anxieux. De plus amples études sont cependant nécessaires pour mieux évaluer la prévalence des troubles de la personnalité dans cette population et pour déterminer en quoi ils pourraient favoriser l'émergence d'un tableau clinique de type PTSD ou d'épisode dépressif majeur.

Dans notre centre d'interventions de crise, les interventions thérapeutiques consistent en premier lieu à favoriser la mobilisation des ressources internes et externes du patient en augmentant la contenance des soins (intensification du nombre d'entretiens, possibilités de passer des nuits dans le service). Le travail avec la famille et avec le réseau tertiaire vise à donner un sens à la souffrance psychique, tout en se dégageant un tant soit peu de l'urgence de la situation sociale, sans toutefois en négliger les enjeux (Bartolomei, Zimmermann, Di Giorgio et ReyBellet, 2012). Il s'agit de préserver une continuité et une cohérence dans les soins entre les différents acteurs impliqués, et de relier les événements actuels avec l'histoire du patient afin de limiter la fragmentation liée au trauma. Ces interventions de crise comprennent classiquement :

- des entretiens individuels infirmiers et médicaux,

- des entretiens de couple et de famille,

- des interventions sociales,

- des interventions groupales centrées sur la médiation,

- un travail de crise, mené conjointement avec les différents interlocuteurs composant le réseau du patient (avocat, médecin généraliste, assistant social).

\section{Prise en soin de type " consultation »}

Lorsque le tableau clinique devient moins aigu, ce premier volet d'interventions débouche le plus usuellement sur une prise en soin de type "consultation ", qui implique une intervention également multidisciplinaire. Celle-ci se distingue de la précédente par sa durée (de quelques mois à plusieurs années) et par les thématiques abordées. D'un point de vue diagnostique, les tableaux de dépression et de syndrome de stress-post traumatique prédominent (Premand et collab., 2013), même si cette population comporte une proportion non négligeable de patients souffrant de troubles psychotiques. Outre les soins habituels que sollicitent ces pathologies, il importe de tenir compte également de manière plus spécifique des facteurs de stress post-migratoires (Laban, Gernaat, Komproe, Schreuders et De Jong, 2004), qui vont s'avérer être des déterminants importants de l'évolution clinique. On retrouve parmi ceux-ci :

- les conditions socioéconomiques rencontrées (conditions de logement, revenu limité). À ce sujet, Porter et Haslam (2005) ont relevé que la précarité du logement, peu ou pas d'opportunité économique (absence d'activité professionnelle) et un conflit persistant dans le pays d'origine influent négativement la santé mentale des réfugiés, 
- les conditions socio-religieuses (possibilité de pratiquer sa religion, sentiment d'être accepté ou au contraire discriminé),

- I'accès à des soins somatiques et psychiques de bonne qualité,

- le maintien d'une cohésion familiale,

- la procédure légale en cours et le sentiment d'insécurité qui y est lié,

- la cohabitation entre différentes communautés.

Pour répondre à ces besoins, outre les interventions de psychiatrie classique, le travail de consultation spécialisé, tout comme celui de crise, va mettre l'accent sur les liens avec les interlocuteurs de référence du patient constituant son réseau. Sont impliqués ordinairement l'avocat responsable de son dossier, le médecin de premier recours, l'infirmier en contact direct avec le patient sur son lieu de vie ainsi que le travailleur social référent pour sa situation (Premand et collab., 2013).

Une autre spécificité du travail concerne la "personne sociale » du requérant d'asile qui, lorsqu'il est débouté, se voit privé de tout accès à une activité rémunérée. II peut en résulter un sentiment d'inutilité et un retrait social accentuant la symptomatologie clinique initiale. Or, certains travaux (Mazur, Chahraoui et Bissler, 2014) ont pointé que les capacités de résilience d'un sujet vont dépendre non seulement de ses défenses psychiques prédominantes mais aussi de la qualité de son entourage et du soutien social dont il bénéficie. Une part de nos interventions vise, à travers l'intégration dans des thérapies groupales ou l'accompagnement dans différentes associations proposant des activités de formation (cours de français, d'informatique, etc.) ou facilitant les liens sociaux (cours de sport, de cusine, etc.), à favoriser la reconstitution d'un réseau social tout en retrouvant un sentiment d'utilité. Ainsi, dans une étude s'intéressant aux besoins exprimés par les patients requérants d'asile, Mc Crone et ses collaborateurs (2005) signalent comme principaux éléments les activités (rémunérées ou non rémunérées), la nourriture et l'accès au logement. Dans une étude similaire, Strijk, van Meijel et Gamel (2011) rapportent pour leur part le soulagement de la détresse psychologique, de l'isolement et l'amélioration des activités quotidiennes. Ces travaux soulignent l'importance de l'étayage social dans la santé mentale des personnes en quête d'asile, et de la possibilité de leur offrir des activités ayant une fonction structurante et un effet valorisant.

\section{Interventions de type "équipe mobile "}

Les interventions de type "équipe mobile " se déploient dans les différents foyers où séjournent les patients requérants d'asile. Elles ont pour objectif un soutien à un sous-groupe de patients souffrant de troubles psychiques graves, pouvant être en difficulté pour maintenir une continuité dans le lien thérapeutique. II s'agit principalement de patients présentant des troubles psychotiques occasionnellement aggravés par une consommation de toxiques et souvent réticents aux soins malgré un haut niveau de souffrance psychique (Maier, Schmidt et Mueller, 2010). Ces patients vont généralement moins consulter que les populations locales (Watters, 2001) ou abréger au maximum la durée de leurs séjours hospitaliers. Nous sommes alors face à une clinique du traumatisme mais aussi de la précarité, concernant des sujets ayant migré seuls, sans réseau primaire, et qui peuvent parfois s'enfermer dans des comportements d'autoexclusion (Furtos, 2011). Les interventions sur leur lieu de vie sont parfois une opportunité de sortir d'une spirale faite d'hospitalisations à répétition et de ruptures rapides de tout traitement ambulatoire. Actuellement, $10 \%$ de la cohorte totale de nos patients requérants d'asile bénéficie de cette modalité de prise en charge, ce qui représente plus de 300 visites par année dans les foyers où résident ces patients. De plus amples recherches sont en cours de développement afin de pouvoir mesurer l'impact d'interventions de ce type sur le taux de ré-hospitalisation de cette population.

\section{Sous-groupe des personnes requérantes d'asile incarcérées}

Il nous semble important de faire également mention du sous-groupe de la population requérante d'asile en détention. Un étude récente réalisée dans le plus important établissement de détention de Genève révélait qu'un peu moins de $90 \%$ des détenus étaient étrangers, 100 nationalités différentes cohabitant dans le même établissement carcéral (Eytan et collab., 2011). Les principales régions représentées étaient l'Afrique du Nord, l'Europe de l'Est et les Balkans ainsi que l'Afrique subsaharienne. Cette surreprésentation de la population 
étrangère doit cependant être traitée avec la plus grande prudence et n'autorise pas pour autant un lien entre criminalité et ressortissants étrangers (Kuhn, 2009)².

Dans l'étude réalisée par Eytan et collaborateurs, $66 \%$ des patients étrangers n'avaient pas de permis de séjour : il s'agissait à $95 \%$ d'hommes, jeunes (âge moyen de 30 ans), et dont $45 \%$ présentaient des plaintes d'ordre psychique. Les auteurs n'ont pas retrouvé d'association significative entre la nationalité et un diagnostic ou un symptôme spécifique des patients, hormis une prévalence légèrement plus élevée dans la consommation d'alcool, de cocaïne et de benzodiazépines chez les ressortissants des pays asiatiques et africains.

La forte prévalence de plaintes psychiques peut être attribuée de manière non spécifique au stress de la détention, mais de manière plus singulière à la faillite du projet migratoire que représente l'incarcération. Ainsi, l'injonction à la réussite que peuvent vivre certains de ces ressortissants de la part de leur famille restée dans leur pays d'origine accroît le sentiment d'échec, dans un contexte d'isolement affectif plus marqué que pour le reste de la population carcérale.

\section{Particularités transculturelles}

Les particularités transculturelles de la population que nous soignons constituent une spécificité importante de notre travail. Pour les diverses ethnies avec lesquelles nous œuvrons, les codes culturels sont différents, et le rapport à la souffrance également. Plus spécifiquement, plusieurs recherches ont pointé la variabilité des modalités d'expression de la souffrance psychique en fonction de l'origine culturelle de chaque sujet (Burnett et Peel, 2001). Certaines cultures vont privilégier le langage du corps pour rendre compte des douleurs de l'âme, d'autres l'agir. Certaines enfin, comme les populations asiatiques, sont connues pour privilégier le bien-être du groupe bien plus que de l'un de ses membres, de telle sorte qu'un individu sera réticent à consulter pour son seul intérêt (Leong et Lau, 2001).

L'expression de la souffrance est la résultante des sensations corporelles, des émotions associées, des modèles explicatifs qu'on y associe et de son accueil/écho par/chez autrui. Tous ces aspects sont influencés par la culture de la personne qui souffre. Par ailleurs, la relation et le degré de proximité socioculturelle entre le clinicien et le patient entrent également en jeu dans ses manifestations (Kleinman, Eisenberg et Good, 1978). La perspective médicale moderne sur les maladies est organisée sur le principe du dualisme corps-esprit et tend à rechercher une origine biologique aux maladies, alors que les personnes malades tendent à penser que leurs problèmes de santé ont plusieurs origines, dont très souvent une cause relationnelle ou psychologique (Helman, 1985; Kirmayer, 2001). Ces points de vue parfois divergents peuvent entraîner des difficultés diagnostiques et de prise en charge importantes, pour les psychiatres en particulier (Mukherjee, Shukla, Woodle, Rosen et Olarte, 1983; Norredam, Garcia-Lopez, Keiding et Krasnik, 2010; Strakowski et collab., 2003).

\section{Conclusion}

Par bien des aspects, les soins institutionnels psychiatriques auprès des patients requérants d'asile représentent un défi permanent pour les cliniciens : l'hétérogénéité des tableaux cliniques rencontrés, la multiplicité des références culturelles accueillies et la variété des parcours migratoires nécessitent un système de soins souple pouvant s'approcher au plus près des besoins du patient, en tenant compte de la singularité de son parcours. Après l'immense avancée qu'ont constitué la reconnaissance et l'exploration de la clinique du trauma auprès de cette population, certains auteurs se questionnent sur le risque réductionniste d'une approche clinique uniquement centrée sur cet angle ( $D^{\prime}$ Halluin, 2006) et sur l'importance croissante de la prise en compte des facteurs de stress

\footnotetext{
${ }^{2}$ En effet, en Suisse, les principales variables explicatives de la criminalité sont le sexe (85\% des condamnation concernant des hommes), le jeune âge et le faible niveau d'éducation. Ces trois caractéristiques sont fortement présentes dans la population migrante, qui est composée surtout d'hommes de faible niveau de formation et plutôt jeunes, de telle sorte que la variable " nationalité » n'a finalement pas d'impact sur la variance de la criminalité. Autrement dit, si on compare le taux de criminalité des étrangers à celui des nationaux en mettant le focus sur la même classe d'âge, de sexe et de niveau de formation, il n'y a plus de différence entre nationaux et étrangers. Ceci est à nuancer si le détenu est en provenance d’un pays en guerre, cas pour lequel on retrouve une augmentation de la violence que l'auteur explique par un effet de déshinibition.
} 
post-migratoires (Silove, Steel, McGorry et Mohan, 1998). À Genève, comme dans différents pays, l'accent est mis sur une approche holistique des soins psychiatriques (Watters, 2001) tenant compte à la fois du vécu traumatique de cette population mais aussi de l'atteinte à la personne " sociale » que peuvent occasionner la combinaison de l'isolement socioprofessionnel et de l'insécurité liée au statut administratif (Vostanis, 2014). Ce second aspect des soins implique une collaboration étroite avec le monde associatif, acteur essentiel dans le travail en réseau auprès de cette population.

De plus amples investigations restent à mener sur les différentes caractéristiques psychopathologiques de cette population ainsi que sur les facteurs susceptibles de les influencer. À cela s'ajoute le fait que les besoins de cette population ne sont pas toujours pris en compte par un modèle de soins occidental souvent à risque de réductionnisme et par une clinique complexe et hétérogène (Watters, 2001). Et ceci alors que nous traversons une époque de durcissement croissant des conditions d'accueil de ce type de population dans plusieurs pays d'Europe.

\section{Remerciements}

De grands remerciements à David Framorando, à Michel Amoros et à Didier Dechoux pour le recueil de certaines données, ainsi qu'à toute l'équipe du secteur pour leur engagement auprès de nos patients.

\section{Bibliographie}

Bartolomei, J., Zimmermann, J., Di Giorgio, S. et Rey-Bellet, P. (2012). Travail de crise avec les patients migrants : de I'injonction à l'agir au retour à la psychopathologie. Psychothérapies, 32(3), 191-197.

Burnett, A. et Peel, M. (2001). Asylum seekers and refugees in Britain: Health needs of asylum seekers and refugees. BMJ : British Medical Journal, 322(7285), 544.

D'Halluin, E. (2006). La mobilisation du clinicien dans les procédures d'asile en France : pratiques et dilemmes. L'Évolution psychiatrique, 71(3), 521-534.

D'Halluin, E. (2009). La santé mentale des demandeurs d'asile. Hommes et migrations. Revue française de référence sur les dynamiques migratoires, 1282, 66-75.

De Coulon, N. et Von Oberbeck Ottino, S. (1999). La crise : stratégies d'intervention thérapeutique en psychiatrie. Paris : Éditions Morin.

Eytan, A., Haller, D., Wolff, H., Cerutti, B., Sebo, P., Bertrand, D. et Niveau, G. (2011). Psychiatric symptoms, psychological distress and somatic comorbidity among remand prisoners in Switzerland. International journal of law and psychiatry, 34(1), 13-19.

Furtos, D. (2011). La précarité et ses effets sur la santé mentale. Le Carnet PSY, 7, 29-34.

Helman, C. (1985). Communication in primary care : the role of patient and practitioner explanatory models. Social science and medicine, 20(9), 923-931.

Holeva, V. et Tarrier, N. (2001). Personality and peritraumatic dissociation in the prediction of PTSD in victims of road traffic accidents. Journal of Psychosomatic Research, 51(5), 687-692.

Hunt, N. et Gakenyi, M. (2005). Comparing refugees and nonrefugees: the Bosnian experience. Journal of anxiety disorders, 19(6), 717-723.

Kirmayer, L. (2001). Cultural variations in the clinical presentation of depression and anxiety: implications for diagnosis and treatment. Journal of Clinical Psychiatry, 62, 22-30.

Kleinman, A., Eisenberg, L. et Good, B. (1978). Culture, illness, and careclinical lessons from anthropologic and cross-cultural research. Annals of internal medicine, 88(2), 251-258.

Kuhn, A. (2013). Comment expliquer la surreprésentation des étrangers dans la criminalité? Vivre Ensemble, HS, 1.

Laban, C., Gernaat, H., Komproe, I. et De Jong, J. (2007). Prevalence and predictors of health service use among Iraqi asylum seekers in the Netherlands. Social psychiatry and psychiatric epidemiology, 42(10), 837-844. 
Laban, C., Gernaat, H., Komproe, I., Schreuders, B. et De Jong, J. (2004). Impact of a long asylum procedure on the prevalence of psychiatric disorders in Iraqi asylum seekers in The Netherlands. The Journal of Nervous and Mental Disorders, 192(12), 843-851.

Leong, F. et Lau, A. (2001). Barriers to providing effective mental health services to Asian Americans. Mental health services research, 3(4), 201-214.

Mc Crone, P., Bhui, K., Craig, T., Mohamud, S., Warfa, N., Stansfeld, S., ... et Curtis, S. (2005). Mental health needs, service use and costs among Somali refugees in the UK. Acta Psychiatrica Scandinavica, 111(5), 351-357.

Mc Coll, H. et Johnson, S. (2006). Characteristics and needs of Asylum Seekers and refugees in contact with London community mental health teams : a descriptive investigation. Social psychiatry and psychiatric epidemiology, 41, 789-795.

Maier, T., Schmidt, M. et Mueller, J. (2010). Mental health and healthcare utilisation in asylum seekers. Swiss medical weekly, 140, w13110.

Mazur, V., Chahraoui, K. et Bissler, L. (2014). Psychopathologies des demandeurs d'asile en Europe, traumatisme et fonctionnement défensif. L'Encephale. http://dx.doi.org/10.1016/j.encep.2013.11.001

Mukherjee, S., Shukla, S., Woodle, J., Rosen, A. et Olarte, S. (1983). Misdiagnosis of schizophrenia in bipolar patients: a multiethnic comparison. The American Journal of Psychiatry, 140(12), 1571-1574.

Norredam, M., Garcia-Lopez, A., Keiding, N. et Krasnik, A. (2010). Excess use of coercive measures in psychiatry among migrants compared with native Danes. Acta Psychiatrica Scandinavica, 121(2), 143-151.

Parker, V. (2002). Connecting relational work and workgroup context in caregiving organizations. The Journal of Applied Behavioral Science, 38(3), 276-297.

Premand, N., Baeriswyl-Cottin, R., Gex-Fabry, M., Coraboeuf, B., Giannakopoulos, P., Eytan, A. et Bartolomei, J. (2013). [Psychiatric care for asylum seekers in Geneva: a multidisciplinary approach for individualized care]. Revue medicale suisse, 9(398), 1664-1666.

Porter, M. et Haslam, N. (2005). Predisplacement and postdisplacement factors associated with mental health of refugees and internally displaced persons: a meta-analysis. JAMA, 294(5), 602-612.

Sanchez Mazas, P. (2011). La construction de l'invisibilité. Genève : Les Éditions IES.

Silove, D., Steel, Z., McGorry, P. et Mohan, P. (1998). Trauma exposure, postmigration stressors, and symptoms of anxiety, depression and post- traumatic stress in Tamil asylum- seekers: comparison with refugees and immigrants. Acta Psychiatrica Scandinavica, 97(3), 175-181.

Sironi, F. (1999). Bourreaux et victimes : psychologie de la torture. Paris : Odile Jacob.

Steel, Z., Chey, T., Silove, D., Marnane, C., Bryant, R. et Van Ommeren, M. (2009). Association of torture and other potentially traumatic events with mental health outcomes among populations exposed to mass conflict and displacement: a systematic review and meta-analysis. JAMA, 302(5), 537-549.

Strakowski, S., Keck Jr, P., Arnold, L., Collins, J., Wilson, R., Fleck, D., ... et Adebimpe, V. (2003). Ethnicity and diagnosis in patients with affective disorders. The Journal of clinical psychiatry, 64(7), 747-754.

Strijk, P., van Meijel, B. et Gamel, C. (2011). Health and social needs of traumatized refugees and asylum seekers: An exploratory study. Perspectives in psychiatric care, 47(1), 48-55.

Vostanis, P. (2014). Meeting the mental health needs of refugees and asylum seekers. The British Journal of Psychiatry, 204(3), 176-177.

Watters, C. (2001). Emerging paradigms in the mental health care of refugees. Social Science and Medicine, 52(11), $1709-1718$

Williams, R. (1999). Personality and posttraumatic stress disorder. Dans W. Yule (dir.), Posttraumatic stress disorder concepts and therapy (p. 93-114). Chichester : John Wiley and Sons.

Alterstice - Revue Internationale de la Recherche Interculturelle, vol. 4, $n^{\circ} 2$ 